\title{
AJCC v8 Stage
}

National Cancer Institute

\section{Source}

National Cancer Institute. A/CC v8 Stage. NCI Thesaurus. Code C132248.

A cancer stage defined according to the AJCC 8th edition criteria. 